\title{
Electroweak boson production and searches for aQGC in CMS
}

\section{Clemencia Mora Herrera*}

Universidade do Estado do Rio de Janeiro

E-mail: clemencia.mora.herreradcern.ch

The production of electroweak bosons is an important part the Standard Model and it can also shed a light on new physics, in the form of anomalous gauge couplings. This poster presents the study of exclusive or quasi-exclusive $\gamma \gamma \rightarrow W^{+} W^{-}$production. The measurement of the process $p p \rightarrow p^{(*)}+W^{+} W^{-}+p^{(*)} \rightarrow p^{(*)}+e^{ \pm} \mu^{\mp}+p^{(*)}$ used an integrated luminosity of $19.7 \mathrm{fb}^{-1}$ of $8 \mathrm{TeV}$ proton-proton collisions at the LHC. Events are selected by requiring the presence of an electron-muon pair with large transverse momentum $p_{\mathrm{T}}\left(\mu^{ \pm} e^{\mp}\right)>30 \mathrm{GeV}$, and no associated charged particles detected from the same vertex. With this analysis the CMS Collaboration has set upper limits on the values of anomalous quartic gauge coupling coefficients for both dim- 6 and dim- 8 EFT operators, giving the most stringent limits to date.

38th International Conference on High Energy Physics 3-10 August 2016

Chicago, USA

* on behalf of the CMS Collaboration 


\section{Introduction}

The process $\gamma \gamma \rightarrow W^{+} W^{-}$occurs at leading order in the Standard Model (SM) through the Feynman diagrams shown in Fig. 1.

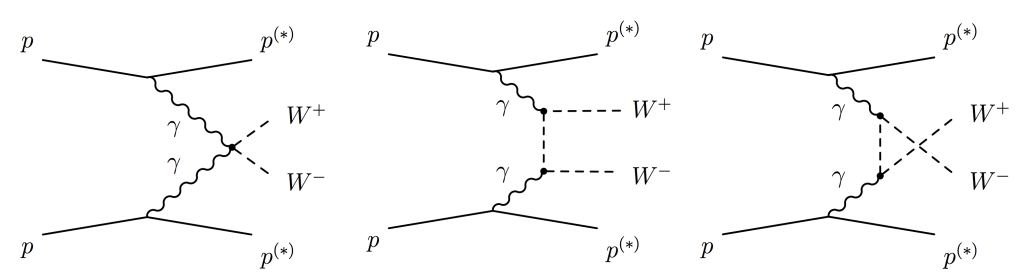

Figure 1: Quartic, $t$-channel and $u$-channel diagrams for $\gamma \gamma \rightarrow W^{+} W^{-}$in the SM.

This is an excellent channel to search for new physics beyond the Standard Model, because of the very low background contribution. Any deviation from the SM may be quantifiable via anomalous quartic gauge couplings (aQGCs) of effective field theory (EFT) operators of dimension-6 [1] or dimension-8 [2].

Dim-6 operators can be constructed with couplings $\frac{a_{0}^{W}}{\Lambda^{2}}$ and $\frac{a_{C}^{W}}{\Lambda^{2}}$, where $\Lambda$ is the scale for new physics. The effective lagrangian terms, originally proposed for LEP are:

$$
\begin{aligned}
& \mathscr{L}_{6}^{0}=-\frac{e^{2}}{8} \frac{a_{0}^{W}}{\Lambda^{2}} F^{\mu v} F_{\mu v} W^{+\alpha} W_{\alpha}^{-}-\frac{e^{2}}{16 c_{W}^{2}} \frac{a_{0}^{Z}}{\Lambda^{2}} F^{\mu v} F_{\mu v} Z^{\alpha} Z_{\alpha} \\
& \mathscr{L}_{c}^{6}=-\frac{e^{2}}{16} \frac{a_{c}^{W}}{\Lambda^{2}} F^{\mu \alpha} F_{\mu \beta}\left(W^{+\alpha} W_{\beta}^{+}\right)-\frac{e^{2}}{16 c_{W}^{2}} \frac{a_{c}^{Z}}{\Lambda^{2}} F^{\mu \alpha} F_{\mu \beta}\left(Z^{\alpha} Z_{\beta}\right) .
\end{aligned}
$$

With the discovery of a light Higgs boson a linear realization of the spontaneously-broken $S U(2) \otimes U(1)$ symmetry of the SM is possible, making dimension- 8 the lowest-order operators where new physics can cause deviations in the purely quartic gauge boson couplings. In this formalism fourteen operators contribute to $\gamma$ couplings, which could also generate a $W W Z \gamma$ vertex. Requiring that the $W W Z \gamma$ vertex vanishes, one can recover a direct relationship between the dim- 8 $f_{M, i} / \Lambda^{4}$ and the dim- $6 \frac{a_{0, C}^{W}}{\Lambda^{2}}$ couplings:

$$
\begin{aligned}
& \frac{a_{0}^{W}}{\Lambda^{2}}=-\frac{4 M_{W}^{2}}{e^{2}} \frac{f_{M, 0}}{\Lambda^{4}}=-\frac{8 M_{W}^{2}}{e^{2}} \frac{f_{M, 2}}{\Lambda^{4}}, \\
& \frac{a_{C}^{W}}{\Lambda^{2}}=\frac{4 M_{W}^{2}}{e^{2}} \frac{f_{M, 1}}{\Lambda^{4}}=\frac{8 M_{W}^{2}}{e^{2}} \frac{f_{M, 3}}{\Lambda^{4}} .
\end{aligned}
$$

Considering the $\gamma \gamma \rightarrow W^{+} W^{-}$diagram with the inclusion of aQGCs, the cross section has a square dependence on the coupling strength. One alternative to regulate the rising of the cross section at high energies is to multiply the $a_{0, C}^{W}$ parameters by a dipole form factor $(\mathrm{FF})$ as in eq. 1.3, where $S_{\gamma \gamma}$ is the energy exchange in the diphoton vertex.

$$
a_{0, C}^{W}\left(W_{\gamma \gamma}^{2}\right)=\frac{a_{0, C}^{W}}{\left(1+\frac{S_{\gamma \gamma}^{2}}{\Lambda_{\text {cutoff }}^{2}}\right)^{2}},
$$

The results in this analysis are studied either using a cutoff energy scale $\Lambda_{\text {cutoff }}=500 \mathrm{GeV}$ or with no cutoff (thus violating unitarity). 


\section{Selection of events for exclusive $\gamma \gamma \rightarrow W W$}

The preselection of events requires an opposite sign muon-electron pair from a common primary vertex, where each lepton with $p_{\mathrm{T}}(l)>20 \mathrm{GeV}$ and $|\eta|<2.4$, and less than 16 additional tracks at vertex. Additional requirements are listed in Tab. 1 with their effect on the expected number of signal and backgrounds events from Monte Carlo (MC) simulation.

\begin{tabular}{l|c|c|c|cccc}
\hline Selection step & Data & $\begin{array}{c}\text { Exclusive } \\
\gamma \gamma \rightarrow W^{+} W^{-}\end{array}$ & $\begin{array}{c}\text { Total } \\
\text { background }\end{array}$ & $\begin{array}{c}\text { Inclusive } \\
\text { diboson }\end{array}$ & Drell-Yan & $\gamma \gamma \rightarrow \tau^{+} \tau^{-}$ & $\begin{array}{c}\text { Other } \\
\text { backgrounds }\end{array}$ \\
\hline Trigger and Preselection & 19406 & $26.9 \pm 0.2$ & $22180 \pm 1890$ & $1546 \pm 15$ & $7093 \pm 75$ & $18.1 \pm 0.8$ & $13520 \pm 1890$ \\
$m\left(\mu^{ \pm} e^{\mp}\right)>20 \mathrm{GeV}$ & 18466 & $26.6 \pm 0.2$ & $21590 \pm 1850$ & $1507 \pm 15$ & $7065 \pm 75$ & $18.1 \pm 0.8$ & $13000 \pm 1850$ \\
Muon and electron identification & 6541 & $22.5 \pm 0.2$ & $6640 \pm 93$ & $1306 \pm 11$ & $4219 \pm 58$ & $12.6 \pm 0.7$ & $1102 \pm 72$ \\
$\mu^{ \pm} e^{\mp}$ vertex with no add. tracks & 24 & $6.7 \pm 0.2$ & $15.2 \pm 2.5$ & $3.7 \pm 0.7$ & $6.5 \pm 2.3$ & $4.3 \pm 0.5$ & $0.7 \pm 0.1$ \\
$p_{\mathrm{T}}\left(\mu^{ \pm} e^{\mp}\right)>30 \mathrm{GeV}$ & 13 & $5.3 \pm 0.1$ & $3.9 \pm 0.5$ & $2.3 \pm 0.4$ & $0.1 \pm 0.1$ & $0.9 \pm 0.2$ & $0.6 \pm 0.1$ \\
\hline
\end{tabular}

Table 1: Number of events passing each cut, for MC the numbers are normalized to $L=19.7 \mathrm{fb}^{-1}$.

\section{Results}

The distributions for data and MC after selection cuts for the number of extra-tracks in events with $p_{\mathrm{T}}\left(\mu^{ \pm} e^{\mp}\right)>30 \mathrm{GeV}$ (right) and for $\mu^{ \pm} e^{\mp}$ transverse momentum in events with zero associated extra-tracks (left) are shown in Fig. 2. Two representative values for anomalous couplings are shown stacked on top of the backgrounds. The first bin in the extra-tracks distribution shows the 13 selected events in data, compatible with SM prediction, which gives a measured cross section of $10.8_{-4.1}^{+5.1} \mathrm{fb}^{-1}$, resulting in a $3.4 \sigma$ significance of the SM signal.
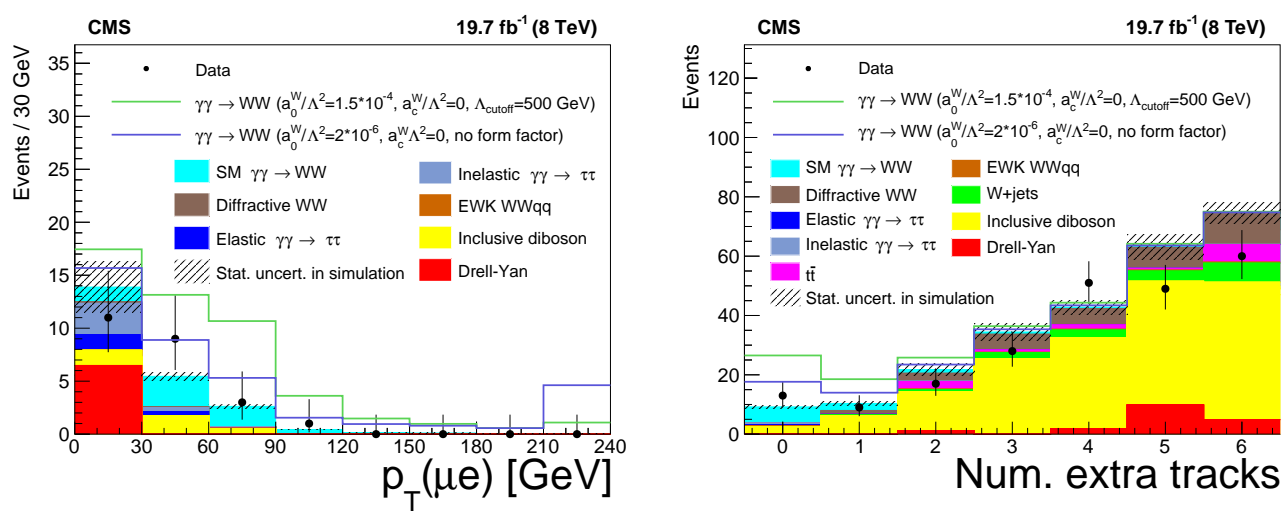

Figure 2: Distributions of the lepton pair $p_{\mathrm{T}}$ for events without extra tracks (left), and extra-tracks multiplicity for events with $p_{\mathrm{T}}\left(\mu^{ \pm} e^{\mp}\right)>30 \mathrm{GeV}$ (right).

The shape of the lepton pair $p_{\mathrm{T}}$ distribution above $30 \mathrm{GeV}$ is used to compute the upper limit on the anomalous cross section [6]. The resulting two dimensional 95\% confidence region in the dimension- 6 aQGC parameter space is shown in Fig. 3 and compared to the $7 \mathrm{TeV}$ result from CMS [3]. The combination of the two measurements is also shown, and the final combined limits for dim- 6 and dim- 8 couplings are: 


$$
\begin{aligned}
-0.9 & <a_{0}^{W} / \Lambda^{2}<0.9\left(\times 10^{-4} \mathrm{GeV}^{-2}\right) \\
-3.6 & <a_{C}^{W} / \Lambda^{2}<3.0\left(\times 10^{-4} \mathrm{GeV}^{-2}\right) \\
-3.4 & <f_{M, 0} / \Lambda^{4}<3.4\left(\times 10^{-10} \mathrm{GeV}^{-4}\right) \\
-14 & <f_{M, 1} / \Lambda^{4}<12\left(\times 10^{-10} \mathrm{GeV}^{-4}\right)
\end{aligned}
$$

for $\Lambda_{\text {cutoff }}=500 \mathrm{GeV}$ and

$$
\begin{aligned}
-1.1<a_{0}^{W} / \Lambda^{2} & <1.1\left(\times 10^{-6} \mathrm{GeV}^{-2}\right) \\
-4.1<a_{C}^{W} / \Lambda^{2} & <4.1\left(\times 10^{-6} \mathrm{GeV}^{-2}\right) \\
-4.2 & <f_{M, 0} / \Lambda^{4}<4.2\left(\times 10^{-12} \mathrm{GeV}^{-4}\right) \\
-16 & <f_{M, 1} / \Lambda^{4}<16\left(\times 10^{-12} \mathrm{GeV}^{-4}\right)
\end{aligned}
$$

without FF, i.e. for $\Lambda_{\text {cutoff }} \rightarrow \infty$.

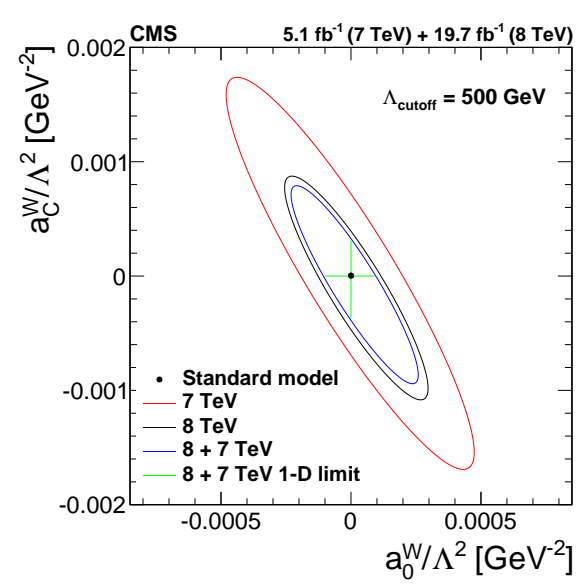

Figure 3: Limits in the dimension-6 aQGC parameters.

\section{Comparisons}

Fig. 4 shows on the left the expected and measured cross sections for various EW boson production modes. Highlighted in yellow is the exclusive $\gamma \gamma \rightarrow W^{+} W^{-}$process which is one of the lowest measured cross-sections at the LHC [5]. The other low-cross section EW production modes also contribute to the searches of anomalous gauge couplings. The plot on the right of Fig. 4 shows the comparison of the obtained limits for the coefficients of dimension- 8 operators of aQGCs in different analyses performed by CMS and ATLAS [4], with the results of the present analysis hightlighted in yellow.
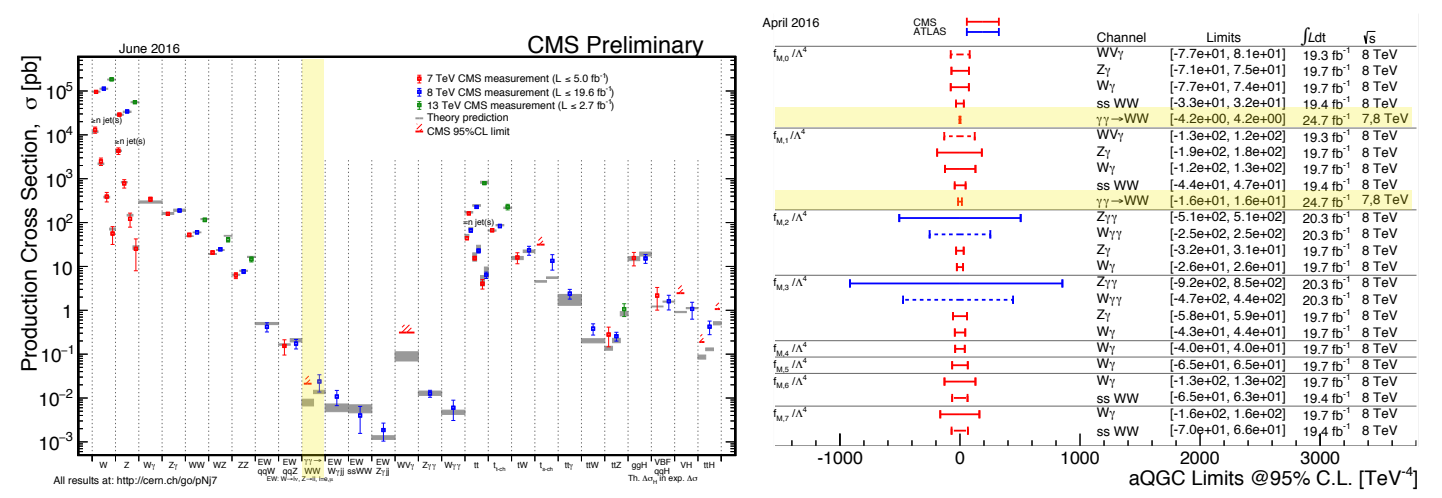

Figure 4: Cross-sections and limits on dim-8 aQGC parameters (no FF) for various LHC measurements.

\section{Conclusions}

The observed yields and kinematic distributions are consistent with the SM prediction, with a combined significance over the background-only hypothesis of $3.4 \sigma$. No significant deviations from the SM are observed, in the $p_{\mathrm{T}}\left(\mu^{ \pm} e^{\mp}\right)$ distribution, and the combined $7+8 \mathrm{TeV}$ limits are interpreted as improved constraints on dimension- 6 and dimension- 8 aQGC parameters. These are the most stringent limits for aQGCs in comparison with other LHC analyses, as shown in the comparison plots. 


\section{References}

[1] G. Belanger and F. Boudjema. Probing quartic couplings of weak bosons through three vectors production at a 500-GeV NLC. Phys. Lett., B288:201-209, 1992. doi: 10.1016/0370-2693(92) 91978-I.

[2] G. Belanger, F. Boudjema, Y. Kurihara, D. Perret-Gallix, and A. Semenov. Bosonic quartic couplings at LEP-2. Eur. Phys. J., C13:283-293, 2000. doi: 10.1007/s100520000305.

[3] CMS Collaboration. Exclusive photon-photon production of muon pairs in proton-proton collisions at $\sqrt{s}=7 \mathrm{TeV}$. JHEP, 01:052, 2012. doi: 10.1007/JHEP01(2012)052.

[4] CMS Collaboration. Limits on anomalous triple and quartic gauge couplings, 2016. URL https://twiki.cern.ch/twiki/bin/view/CMSPublic/ PhysicsResultsSMPaTGC.

[5] CMS Collaboration. Summaries of cms cross section measurements, 2016. URL https: //twiki.cern.ch/twiki/bin/view/CMSPublic/PhysicsResultsCombine.

[6] CMS Collaboration. Evidence for exclusive $\gamma \gamma \rightarrow W^{+} W^{-}$production and constraints on anomalous quartic gauge couplings at $\sqrt{s}=7$ and $8 \mathrm{TeV}$. JHEP, 08:119, 2016. doi: 10.1007/JHEP08(2016)119. 